\title{
Kinesin family member 23 (KIF23) contributes to the progression of bladder cancer cells in vitro and in vivo
}

\author{
Dong-wei $\mathrm{YAO}^{1,2, *}$, Qun SONG ${ }^{2,4}$, Xiao-zhou $\mathrm{HE}^{1, *}$ \\ ${ }^{1}$ Department of Urology, The Third Affiliated Hospital of Soochow University, Changzhou, Jiangsu, China; ${ }^{2}$ Department of Urology, The Second \\ People's Hospital of Lianyungang, Lianyungang, Jiangsu, China \\ ${ }^{*}$ Correspondence: xiaozhouhe123@163.com \\ \#Contributed equally to this work.
}

Received August 3, 2020 / Accepted October 27, 2020

\begin{abstract}
The aim of our study was to detect the expression of KIF23 in human bladder cancer tissues and to assess the potential role of KIF23 in bladder cancer progression. The expression of KIF23 and the correlation with bladder cancer patients were explored using the TCGA database. Additionally, IHC assays were also performed to detect KIF23 expression in 95 bladder cancer tissues and corresponding non-tumor tissues collected in our hospital. Colony formation, MTT, and flow cytometry (FCM) assays were performed to detect its effects on bladder cancer cell proliferation and apoptosis, respectively. An animal model was developed to found the effects of KIF23 on tumor growth in mice. Data showed that the KIF23 expression was upregulated in human bladder cancer tissues. The expression of KIF23 was correlated with the prognosis and clinicopathological features, including T stage $(\mathrm{p}=0.022)$ and recurrence $(\mathrm{p}=0.020)$, of bladder cancer patients. KIF23 depletion inhibited the proliferation of bladder cancer cells, stimulated apoptosis, and suppressed tumor growth in mice. We demonstrated the involvement of KIF23 in bladder cancer progression and provided a promising therapeutic target for the treatment of bladder cancer.
\end{abstract}

Key words: Kinesin family member 23 (KIF23), bladder cancer, proliferation, apoptosis

Bladder cancer is a common type of cancer in the urinary system, with a high incidence worldwide. According to recent research, about 80,470 new bladder cancer cases could be identified, and the estimated new deaths were 17,670 cases in the United States in 2019 [1]. The common treatment for non-muscle invasive bladder cancer (NMIBC) is a transurethral resection of bladder tumor (TURBT) followed by intravesical therapy. For patients with muscle invasive bladder cancer (MIBC), radical cystectomy with urinary diversion is recommended, and those who have pelvic lymph node metastasis or distant metastasis also need radiotherapy and/ or chemotherapy. For metastasized bladder cancer patients, the outcome is still poor, with a median survival time of approximately 14 months [2]. Emerging immune checkpoint inhibitors (ICB) bring hope to patients with metastatic bladder cancer. However, only $13-21 \%$ of patients with metastatic bladder cancer benefited from ICB [3-5]. Due to the high incidence and the nature of its management, bladder cancer has become one of the most expensive cancers in China. To combat this disease more specifically, there is still an urgent need to develop new promising therapeutic targets.
Kinesin family member 23 (KIF23), a human homolog of mouse Kif23, is a critical motor protein involved in multiple cellular processes such as motility, cytokinesis, and ciliogenesis [6, 7]. In HeLa cells, the depletion of KIF23 led to the formation of multinucleated cells due to the defects of cytokinesis [8]. A previous study also indicated that KIF23 could drive the movement of the microtubule so that to affect the microtubule-dependent functions $[6,9]$. Additionally, in neuroblastoma cells, the ablation of KIF23 also led to an obvious increase in axon length [10].

Notably, the wide involvement of KIF23 in the progression of multiple types of tumors was revealed [11, 12]. KIF23 was found abnormally expressed in several types of cancers, including lung cancer, breast cancer, and gastric cancer [11-13]. KIF23 contributed to gastric cancer by promoting cell proliferation [13]. Additionally, another study reported a higher KIF23 expression in glioma tissues compared to normal brain tissues [14]. Though the wide effects of KIF23 on cancer progression, the potential role of KIF23 in the progression and development of bladder cancer is still unclear. 
In this study, we explored the possible involvement of KIF23 in the progression of bladder cancer. We found the high expression of KIF23 in human bladder cancer tissues, and its expression correlated with the prognosis and clinicopathological features of patients with bladder cancer. We further demonstrated that KIF23 promoted the proliferation, stimulated the apoptosis of bladder cancer cells, which was confirmed in mice. In conclusion, we confirmed KIF23 as a promising therapeutic target for the treatment of bladder cancer.

\section{Materials and methods}

Antibodies, primers, and shRNA plasmids. Anti-KIF23 (for IHC assays, 1:200 dilution, ab235955, Abcam, Cambridge, UK; for immunoblot assays, 1:2000 dilution, ab174304, Abcam, Cambridge, UK), anti-GAPDH (1:2000 dilution, ab9484, Abcam, Cambridge, UK).

The quantitative PCR primer sequences of KIF23 are as follows: forward, 5'-AGACAGAAGGCGAGGGATG-3' and reverse, 5'-GGAGACGAATTGGTGGTGC-3'; the quantitative PCR primer sequences of GAPDH are as follows: 5'-CGACCACTTTGTCAAGCTCA-3' and 5'-GGTTGAGCACAGGGTACTTTATT-3'. KIF23 shRNA plasmids (the shRNA sequence targeting KIF23 was as follows: 5'-GGTAGATCTTGCTGGAAGT-3') were purchased from Addgene plc.

Human tissue samples and analysis. The 95 human bladder cancer tissues and corresponding non-tumor tissues in this study were collected from the patients receiving surgical treatment in our hospital. The clinicopathological characteristics, such as patient age, gender, $\mathrm{T}$ stage, tumor grade, lymph node metastasis, recurrence was listed in Table 1.

To explore the possible correlations between the expression levels of KIF23 and bladder cancer progression, immunohistochemical (IHC) assays were performed on formalin-fixed, paraffin-embedded bladder cancer tissues. In brief, tissue sections $(4 \mu \mathrm{m})$ were deparaffinized for $10 \mathrm{~min}$ in xylene, then rehydrated with ethanol. After antigen retrieval, $3 \% \mathrm{H}_{2} \mathrm{O}_{2}$ was added to the sections and incubated for 15 minutes at room temperature to block endogenous peroxidase activity. Slides were incubated with KIF23 antibodies at room temperature for 2 hours. Subsequently, the sections were incubated with biotinylated secondary antibody for another 1.5 hours, and diaminobenzidine was used as a chromogen substrate.

KIF23 was found mainly located in the nucleus of bladder cancer tissues. The score methods were as follows. In brief, the proportion of positive stained cells was graded as follows: $1=0-25 \%$ stained cells; $2=26-50 \%$ stained cells; 3 $=51-75 \%$ stained cells; $4=76-100 \%$ stained cells [15]. The staining intensity was evaluated on a score of 0 (no staining), 1 (low-level staining), 2 (modest-level staining), and 3 (highlevel staining). The final KIF23 staining score = the intensity score $\times$ the percentage score, the expression levels of KIF23 were defined as follows: a final staining score less than 6 was considered as a low expression, while a staining score of 7 or more was thought as a high expression [16].

Cell culture and transfection. The human bladder cancer cell lines, T24 and 5637, were bought from ATCC. T24 cells were incubated in Dulbecco's modified essential medium (DMEM), supplemented with $10 \%$ fetal bovine serum (FBS, Gibco, CA, USA), in a 5\% $\mathrm{CO}_{2}$ incubator. 5637 cells were cultured in RPMI 1640 medium containing $10 \%$ fetal bovine serum (FBS, Gibco, CA, USA), in a $5 \% \mathrm{CO}_{2}$ incubator. The KIF23 shRNA plasmids were transfected into both T24 and 5637 cells using the Lipofectamine 2000 reagent (11668019, Invitrogen, CA, USA) following the manufacturer's instructions. KIF23 stably depleted T24 cells were screened through its shRNA lentivirus infection and used for the in vivo tumor growth assays.

Quantitative PCR assays. Trizol (15596026, Invitrogen, CA, USA) was used to extract total RNA from human bladder cancer cells according to the manufacturer's protocol. Then the RNA was reverse-transcribed by M-MLV reverse transcriptase (M1701, Promega, Wisconsin, USA).

Total mRNA was reverse transcribed to produce cDNA by the cDNA synthesis system. Quantitative PCR was performed using SYBR Ex Taq kit (638319, Takara, Japan), and KIF23 expression level was normalized to the expression of GAPDH as an internal control.

Immunoblot assays. Bladder cancer cells or tissues were lysed by RIPA Buffer (9800, Cell Signaling, Danvers, MA). Then SDS-PAGE was performed. After the transfer onto

Table 1. The correlations between KIF23 expression and the clinicopathological features of 95 patients with bladder cancer.

\begin{tabular}{|c|c|c|c|c|c|}
\hline \multirow[b]{2}{*}{ Feature } & \multirow[b]{2}{*}{$\underset{\mathrm{n}=95}{\text { All }}$} & \multicolumn{2}{|c|}{ KIF23 expression } & \multirow[b]{2}{*}{$\chi^{2}$} & \multirow[b]{2}{*}{ p-value } \\
\hline & & $\begin{array}{c}\text { Low } \\
n=26\end{array}$ & $\begin{array}{l}\text { High } \\
n=69\end{array}$ & & \\
\hline Age (year) & & & & 2.229 & 0.135 \\
\hline$<55$ & 36 & 13 & 23 & & \\
\hline$\geq 55$ & 59 & 13 & 46 & & \\
\hline Gender & & & & 1.803 & 0.179 \\
\hline Male & 63 & 20 & 43 & & \\
\hline Female & 32 & 6 & 26 & & \\
\hline Tumor stage & & & & 5.235 & $0.022^{*}$ \\
\hline $\mathrm{T} 2$ & 44 & 17 & 27 & & \\
\hline T3/T4 & 51 & 9 & 42 & & \\
\hline Tumor grade & & & & 0.839 & 0.360 \\
\hline Low & 23 & 8 & 15 & & \\
\hline High & 72 & 18 & 54 & & \\
\hline Lymph node metastasis & & & & 2.723 & 0.099 \\
\hline Yes & 25 & 10 & 15 & & \\
\hline No & 70 & 16 & 54 & & \\
\hline Recurrence & & & & 5.414 & $0.020^{*}$ \\
\hline Yes & 44 & 7 & 37 & & \\
\hline No & 51 & 19 & 32 & & \\
\hline
\end{tabular}


polyvinylidene fluoride (PVDF) membranes, membranes were blocked with $5 \%$ skim milk in TBST at room temperature for $2 \mathrm{~h}$. After that, the proteins were incubated with the primary antibodies for the detection of KIF23 and GAPDH overnight at $4{ }^{\circ} \mathrm{C}$. Then the PVDF membranes were incubated with HRP-conjugated secondary antibodies for 1 hour. Signals were detected by an ECL kit (E411-03, Vazyme, Nanjing, China).

Colony formation assays. The bladder cancer cells were transfected with control or KIF23 shRNA plasmids for $48 \mathrm{~h}$ and then cultured into a 6-well culture plate (200 cells/ well). The medium was replaced with fresh medium every 3 days. After two weeks, cells were fixed with PFA for $30 \mathrm{~min}$ at room temperature and stained with $0.2 \%$ crystal violet at room temperature for $30 \mathrm{~min}$, then washed with PBS. The plates were photographed and the number of colonies was manually counted.

MTT assays. The bladder cancer cells were plated into 96-well plates with a density of approximately 3000 cells/well, then transfected with control or KIF23 shRNA plasmids and incubated for 24 hours. After that, the cells were incubated with $20 \mu \mathrm{l}$ MTT reagent for $4 \mathrm{~h}$ at room temperature following the removal of the culture medium. Then $150 \mu \mathrm{l}$ dimethyl sulfoxide (DMSO) was added into each well to extract the stained cells and the OD value was measured with a microplate reader at $570 \mathrm{~nm}$ wavelength.

Cell apoptosis assays. For cell apoptosis assays, bladder cancer cells were stained with annexin V-FITC and PI for 20 min at room temperature. Then cells were analyzed using a FACS Calibur flow cytometer.

Tumor growth assays. All animal assay processes were approved by the Institutional Animal Care and Use Committee (IACUC) of our hospital. T24 cells were stably infected with control or KIF23 shRNA lentivirus. Subse- quently, about $1 \times 10^{6} \mathrm{~T} 24$ cells infected with the indicated lentivirus were subcutaneously implanted into athymic nude mice (Control group $\mathrm{n}=4$, KIF23 shRNA group $\mathrm{n}=4$ ). After 15 days, tumors began formation, and the tumor volume was measured every 3 days. After 30 days, tumors were taken out and photographed, and the tumor growth curves were calculated.

Statistics. GraphPad 6.0 software was used for statistical analysis in this study. All results in this study were represented as mean $\pm \mathrm{SD}$. The correlations between clinical features of bladder cancer patients and KIF23 expression levels were analyzed using $\chi^{2}$ analysis. Student's t-test was used for statistical comparisons. ${ }^{*}$ indicated $\mathrm{p}<0.05$, which was also considered a statistically significant difference.

\section{Results}

KIF23 expression was enhanced in bladder cancer tissues and correlated with the prognosis of bladder cancer. To evaluate the expression of KIF23 in human bladder cancer tissues, we searched the expression information of KIF23 in bladder cancer tissues according to the TCGA database using the GEPIA web server. We found that the expression of KIF23 in bladder cancer was at a high level compared to non-tumor tissues (Figure 1A). We then assessed the survival information of patients with bladder cancer according to two sets of patients with different expression levels of KIF23. Interestingly, we found the high expression of KIF23 was correlated with disease-free survival rates of patients with bladder cancer, suggesting the correlation between KIF23 and the prognosis of bladder cancer patients (Figure 1B). Therefore, data in the TCGA database provided evidence that KIF23 was highly expressed in human bladder cancer tissues and correlated with the prognosis.
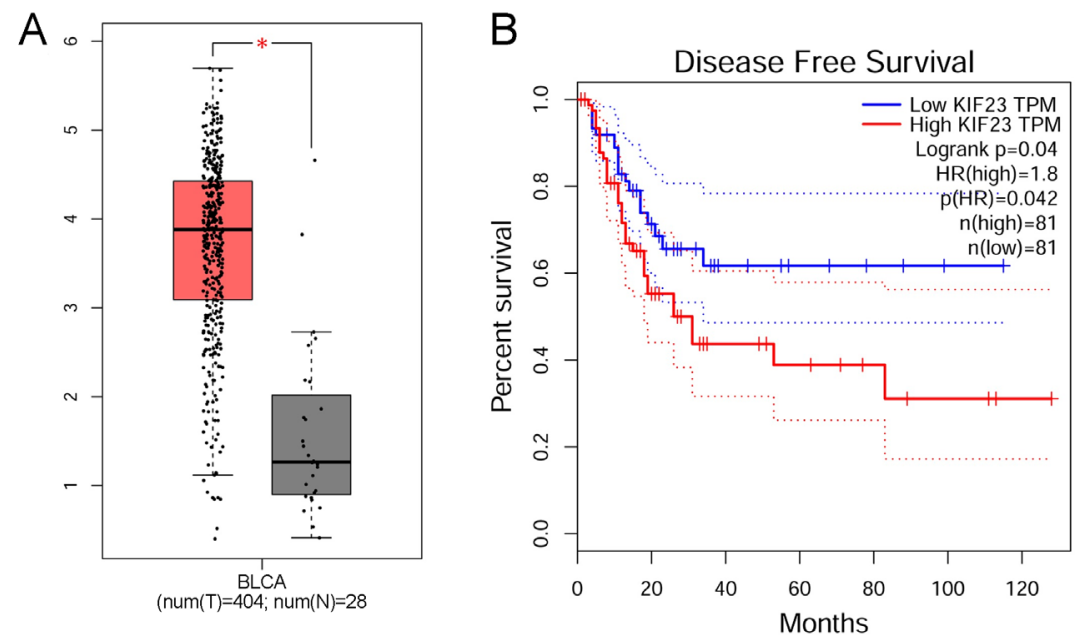

Figure 1. KIF23 expression was enhanced in bladder cancer tissues and correlated with the prognosis of patients with bladder cancer. A) KIF23 mRNA level (using $\log 2(T P M+1)$ for log-scale) in bladder cancer tissues was upregulated according to the TCGA database (GEPIA web server [29]). B) KIF23 expression was correlated with the disease-free survival rates of two sets of bladder cancer patients, according to the TCGA database. 
The expression of KIF23 was associated with the clinicopathological features of bladder cancer patients. Subsequently, we performed the IHC assays to further detect the expression levels of KIF23 in a total of 95 bladder cancer tissues and corresponding normal tissues collected in our hospital. According to the staining results, we noticed KIF23 was mainly located in nuclei of bladder cancer tissues (Figure 2A). Importantly, the expression of KIF23 was obviously higher than that in the corresponding normal tissues, consistent with the previous results (Figures 2A, 2B).

We then performed a clinicopathological analysis to explore the correlation between KIF23 expression and clinical features of the 95 bladder cancer patients. Patients were divided into KIF23 high expression and low expression according to the staining results, we found $26(27.3 \%)$ patients showed low expression, and the remaining 69 (72.7\%) exhibited high KIF23 expression (Table 1).

The clinical features, including patient age, gender, T stage, tumor grade, lymph node metastasis, and recurrence, were analyzed (Table 1). We found there were no obvious correlations between KIF23 expression and patient age $(p=0.135)$, gender $(\mathrm{p}=0.179)$, tumor grade $(\mathrm{p}=0.036)$, and lymph node metastasis $(\mathrm{p}=0.099$, Table 1$)$. Notably, the expression of KIF23 was significantly correlated with $T$ stage $(p=0.022)$ and recurrence $(\mathrm{p}=0.020$, Table 1$)$ of bladder cancer patients. We, therefore, indicated the high expression of KIF23 in human bladder cancer tissues and confirmed the correlations between its expression and clinical pathological characteristics of bladder cancer patients.

Knockdown of KIF23 suppressed the proliferation and stimulated the apoptosis of bladder cancer cells in vitro. To further assess the involvement of KIF23 in the progression of bladder cancer, we used the shRNA plasmids targeting
KIF23 to deplete its expression in human bladder cancer cell lines, T24 and 5637. The silencing efficiency was confirmed by quantitative PCR assays (Figure 3A) and immunoblot assays (Figure 3B), respectively, in T24 and 5637cells, and the mRNA and protein levels were all obviously decreased after KIF23 depletion.

Subsequently, we performed MTT assays and colony formation assays to explore the effects of KIF23 on the proliferation of T24 and 5637 cells. We noticed the ablation of KIF23 led to the decrease of colony numbers in T24 and 5637 cells, confirmed by colony formation assays (Figure 4A). Similarly, through MTT assays, we found the OD value at $570 \mathrm{~nm}$ wavelength was obviously decreased in T24 and 5637 cells after KIF23 depletion, suggesting the defects in cell proliferation (Figure 4B). We further detected the effects of KIF23 on cell apoptosis in T24 and 5637 cells transfected control or KIF23 shRNA plasmids, through the flow cytometry (FCM) assays. Interestingly, we found the depletion of KIF23 increased the percentage of apoptotic cells in both T24 and 5637 cells, respectively, suggesting the promotion of cell apoptosis (Figure 4C). Collectively, we demonstrated the depletion of KIF23 could lead to proliferation defects and stimulated the apoptosis of bladder cancer cells in vitro.

KIF23 promoted tumor growth of bladder cancer cells in mice. To further confirm the previous in vitro data, we explored whether KIF23 promoted tumor growth of bladder cancer cells using an animal model. Briefly, T24 cells were infected with the control or KIF23 shRNA lentivirus and subcutaneously injected into nude mice. After 15 days, the tumor began formation and tumor volume was measured every 3 days. After 30 days, all tumors were isolated, photographed, and the animals were sacrificed. The tumor growth curves are exhibited in Figure 5A, left. The representative
A

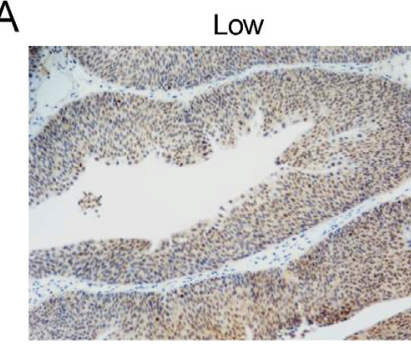

D

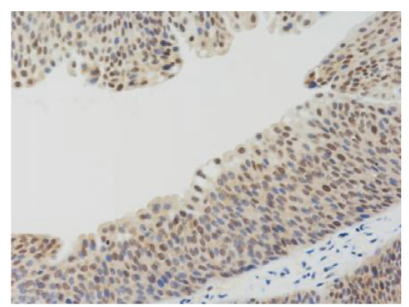

B

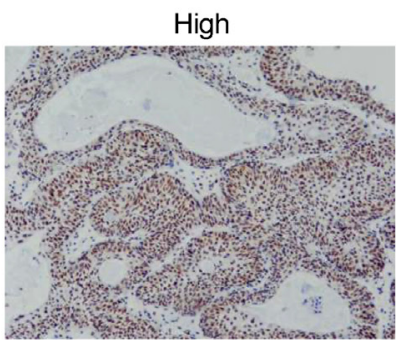

E

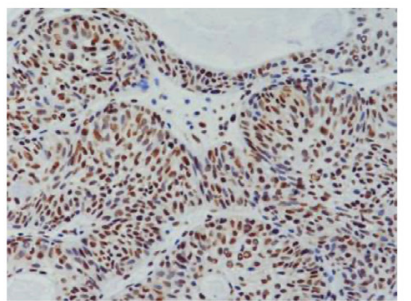

C

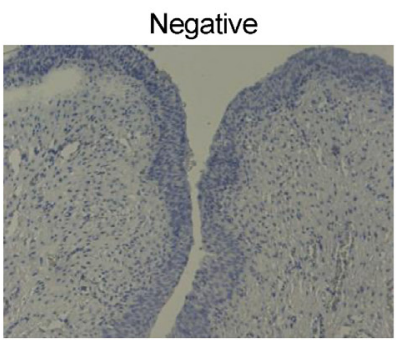

$\mathrm{F}$

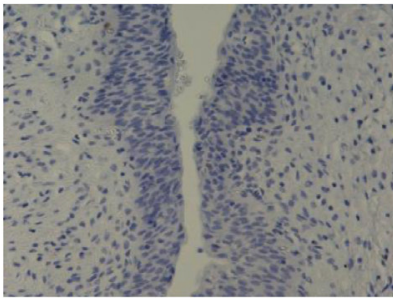

Figure 2. KIF23 expression was upregulated in human bladder cancer tissues. A, B, D, E). Immunohistochemical (IHC) assays were performed, and the representative photographs of KIF23 staining in human bladder cancer tissues were taken and shown (low and high expression, $100 \times$ and $200 \times$ magnification, respectively). C, F) IHC assays were performed and confirmed the negative expression of KIF23 in the corresponding normal tissues (100× and $200 \times$ magnification, respectively). 
A
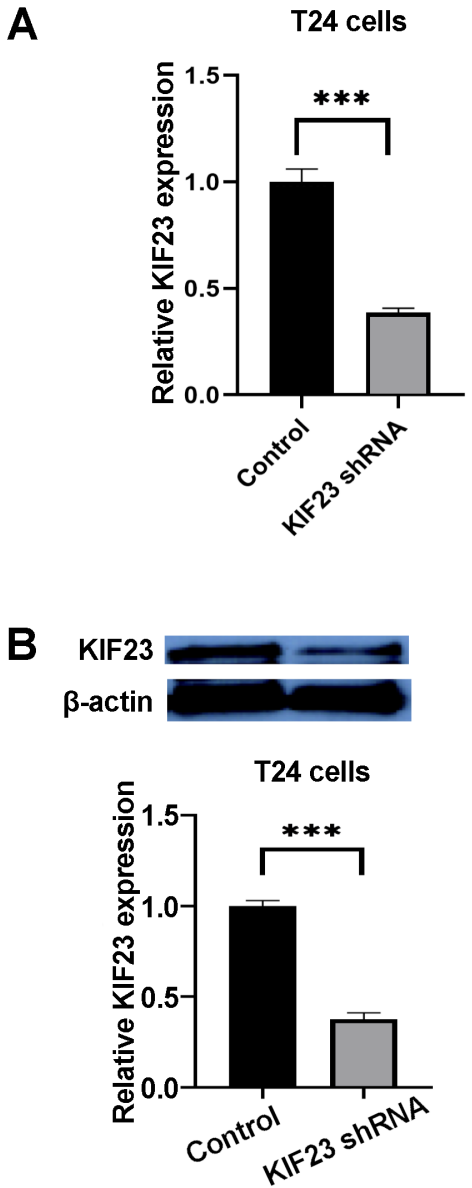

5637 cells

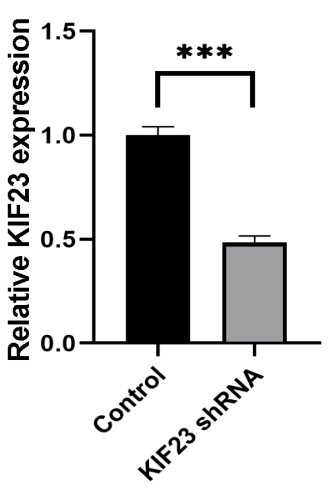

Figure 3. KIF23 expression was decreased in both T24 and 5637 human bladder cancer cells caused by the transfection of its shRNA plasmids. A) Quantitative PCR assays revealed the remarkably decrease mRNA levels of KIF23 following the transfection of its shRNA plasmids in T24 and 5637 cells, respectively. B) Immunoblot assays revealed the efficient inhibition of KIF23 expression after the transfection of its shRNA plasmids in both T24 and 5637 cells. Results are presented as mean $\pm \mathrm{SD},{ }^{*} \mathrm{p}<0.05$

images of tumors are shown in Figure 5A, right. Consistent with our hypothesis, the volume of tumors in KIF23 depletion groups was significantly decreased than that in control groups (Figure 5A). We further performed immunoblot assays to detect KIF23 expression in tumor tissues from control and KIF23 depletion mice. We found KIF23 expression levels were all decreased in KIF23-depleted tumor tissues, consistent with the previous data (Figure 5B). Collectively, we reported KIF23 promoted tumor growth of bladder cancer cells in vivo.

\section{Discussion}

Around $80 \%$ of cases with NMIBC will relapse and about $30 \%$ of patients will progress into MIBC [17]. For NMIBC patients, intravesical chemotherapy and lifelong surveillance cystoscopy are necessary, which makes bladder cancer treatment very expensive in many countries. The recommended treatment options for patients with MIBC include radical cystectomy and platinum-based neoadjuvant or adjuvant chemotherapy [18]. However, there are still many patients present with advanced and metastatic bladder cancer which are associated with extremely low survival rates. ICB bring dawn to patients who are nonresponsive to platinum-based therapy, with an ORR of around 20\% [19, 20]. Indeed, comprehensive treatment strategies for bladder cancer can improve its prognosis but they also impose a heavy economic burden on patients and society. Thus, developing novel and promising therapeutic targets for the targeted therapy of bladder cancer is quite a requirement. In this study, we found the significantly high expression of a kinesin, KIF23, in bladder cancer tissues, according to the data in the TCGA database and our results in IHC assays. Furthermore, we noticed the expression of KIF23 was dramatically correlated with the prognosis and clinicopathological features of bladder cancer patients. Our data, therefore, provided evidence that KIF23 could act as a promising bladder cancer therapeutic target. 
A

A

T24

5637
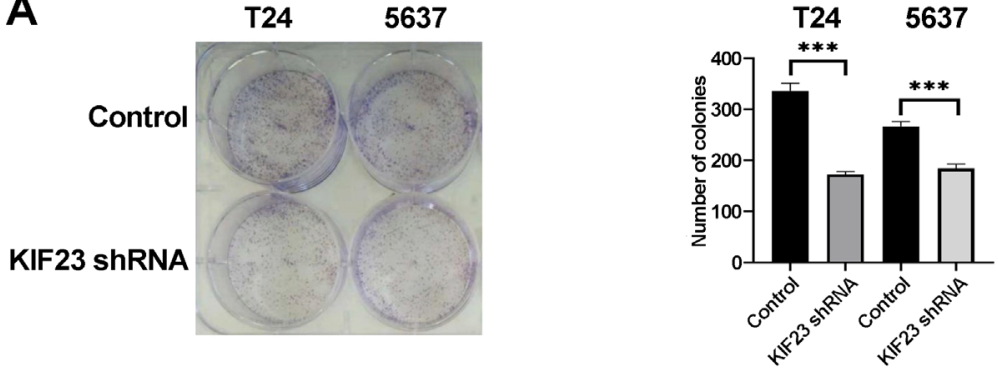

B

T24

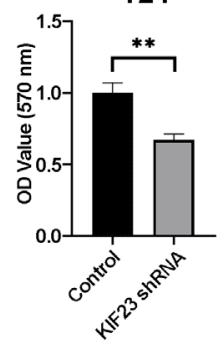

5637

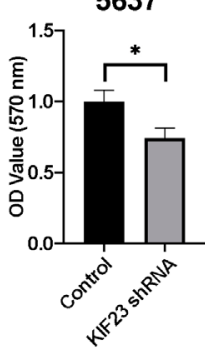

C

Control KIF23 shRNA
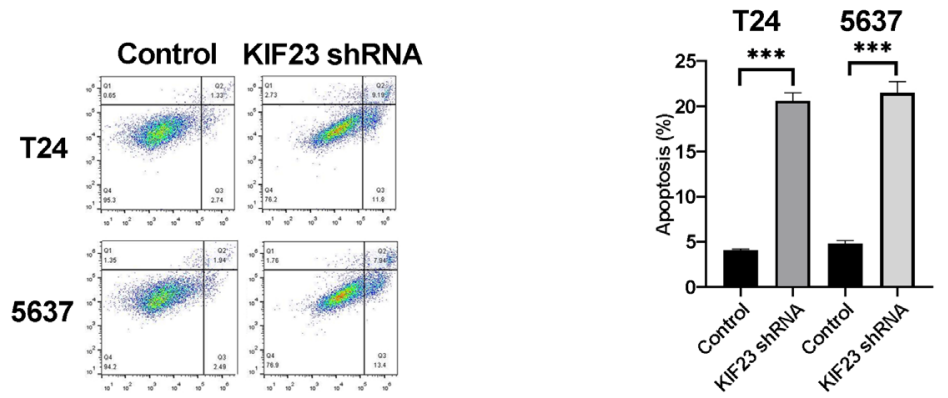

Figure 4. KIF23 affects the proliferation and apoptosis of bladder cancer cells in vitro. A) T24 and 5637 cells transfected with control or KIF23 shRNA plasmids, colony formation assays were conducted, and the number of colonies was counted. B) The results of MTT assays showed a decrease OD value at $570 \mathrm{~nm}$ wavelength after KIF23 depletion. C) Flow cytometry assays were performed using T24 and 5637 cells transfected with control or KIF23 shRNA plasmids, and the effects of KIF23 on cell apoptosis were detected. Results are presented as mean \pm SD, ${ }^{*} \mathbf{p}<0.05$

Subsequently, through MTT assays and colony formation assays, we demonstrated that KIF23 promoted the proliferation of bladder cancer cells in vitro. The FCM assays further confirmed KIF23 affected the apoptosis of bladder cancer cells, and the in vivo assays confirmed the previous data. We, therefore, thought KIF23 affected the progression of bladder cancer via the regulation of cell proliferation and apoptosis. Similarly, the effects of KIF23 on cancer progression and development have been widely revealed [11-14]. KIF23 overexpression was correlated with the clinical outcome in patients with lung cancer [21]. Additionally, KIF23 was identified as a novel prognostic factor in patients with hepatocellular carcinoma (HCC) [22]. Similarly, another study also indicated that KIF23 could promote the proliferation of glioma cells [14]. These studies, together with our findings, suggested the wide effects of KIF23 on cancer progression.

As a key motor protein, KIF23 regulated multiple cellular processes such as mitosis [23]. KIF23 is known to be involved in the regulation of bundling and transport of microtubules to explicit intracellular locations [13, 24]. Furthermore, KIF23 therefore might mediate the migration and mitosis, particularly cytokinesis, of tumor cells [6]. In this study, we noticed KIF23 promoted the progression of bladder cancer via regulating cell proliferation and apoptosis, and thought KIF23 affected this process via the effects on mitosis of tumor cells, and the precise molecular mechanism needs further study. In addition, since the regulation of KIF23 on the microtubule, we next should detect whether KIF23 could affect the migration and invasion of bladder cancer cells in vitro.

Except for KIF23, the effects of other kinesins have been widely revealed [25]. KIF11 could enhance the self-renewal capacity of breast cancer via the Wnt pathway [26]. Another study demonstrated that KIF18B contributed to the proliferation of pancreatic cancer cells via activating CDCA8 expression [27]. Additionally, KIF2A expression was correlated with the tumor stages and prognosis of patients with non-small cell lung cancer [28]. These studies, together with our findings, confirmed the key roles of kinesins in cancer 
A
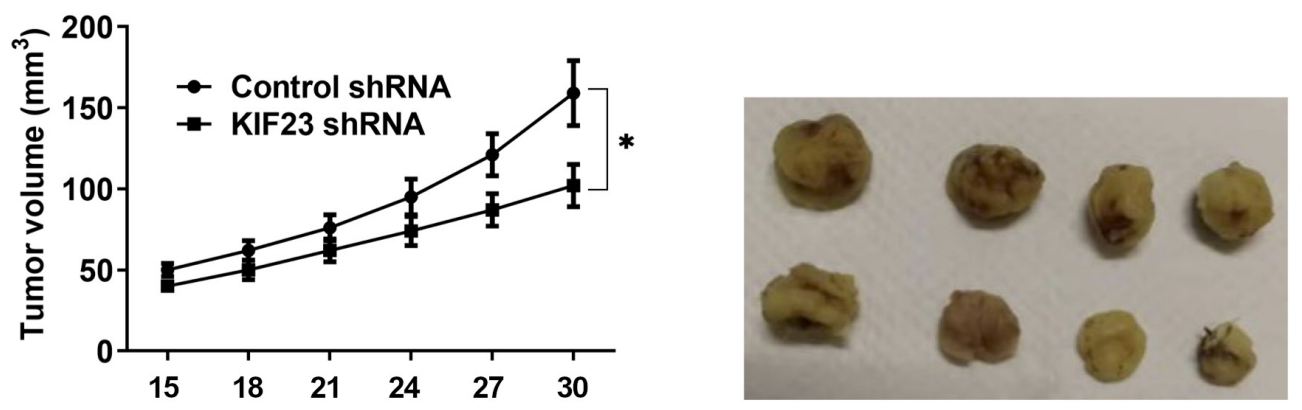

Control

Days after injection

B
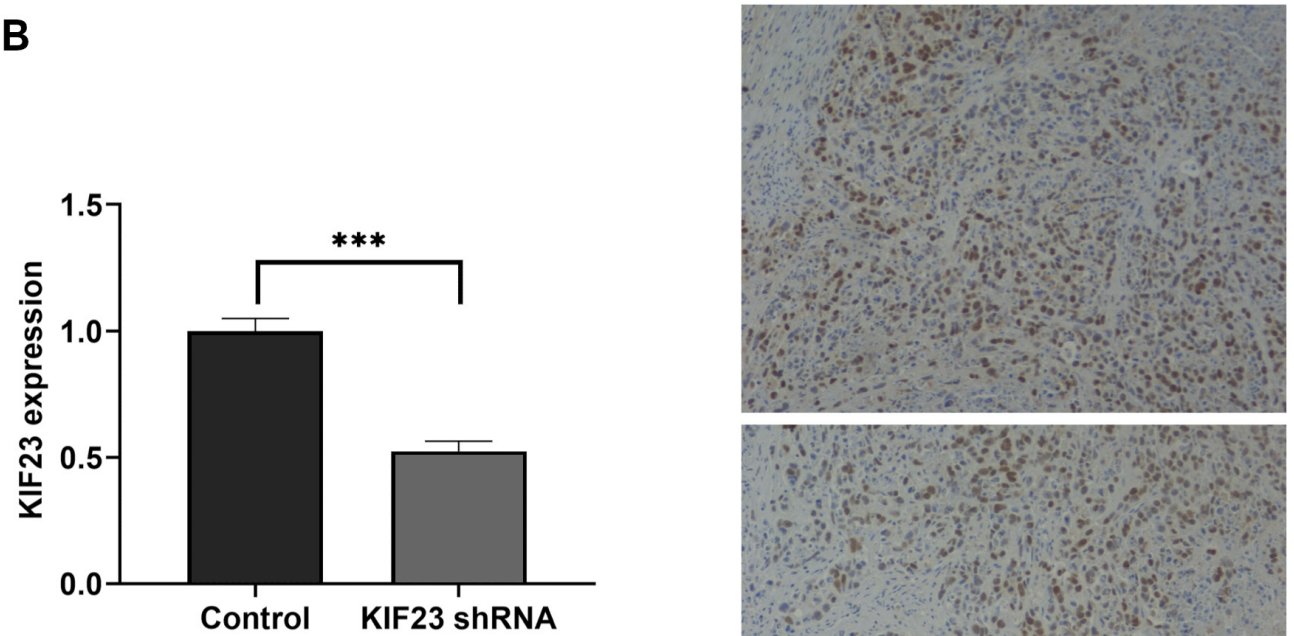

Control

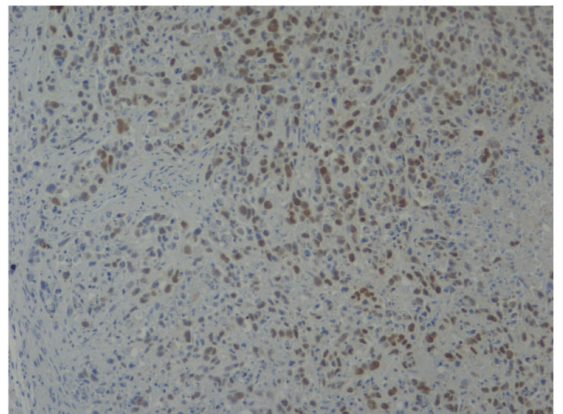

KIF23 shRNA

Figure 5. KIF23 stimulates tumor growth of bladder cancer cells in mice. A) T24 cells infected with control or KIF23 shRNA lentivirus were subcutaneously implanted into nude mice. After 15 days, tumors began formation, and the volume of the tumor was measured every 3 days. After 30 days, all tumors were isolated (right) and the growth curves were analyzed (left) according to the average volume of tumors in KIF23 depletion and control groups. B) Immunoblot assays revealed the expression levels of KIF23 in control or KIF23 depletion tumors isolated from mice (left). IHC assays were performed, and the representative photographs of KIF23 staining in mice bladder cancer tissues were taken and shown (200× magnification). Results are presented as mean $\pm \mathrm{SD},{ }^{\star} \mathrm{p}<0.05$

progression. Therefore, finding effective inhibitors might be a powerful manner for cancer treatment.

In conclusion, we found a high mRNA level of KIF23 in human bladder cancer tissues. The expression of KIF23 was related to the prognosis and clinicopathological features of patients with bladder cancer. We also confirmed KIF23 affected the proliferation and apoptosis of bladder cancer cells in vitro, and affected tumor growth in mice. Therefore, we demonstrated the involvement of KIF23 in bladder cancer progression and thought of KIF23 as a promising bladder cancer therapeutic target.

\section{References}

[1] GOLLA V, LENIS AT, FAIENA I, CHAMIE K. Intravesical Therapy for Non-muscle Invasive Bladder Cancer-Current and Future Options in the Age of Bacillus Calmette-Guerin Shortage. Rev Urol 2019; 21: 145-153.

[2] HINDUPUR SV, SCHMID SC, KOCH JA, YOUSSEF A, BAUR EM et al. STAT3/5 Inhibitors Suppress Proliferation in Bladder Cancer and Enhance Oncolytic Adenovirus Therapy. Int J Mol Sci 2020; 21: 1106. https://doi.org/10.3390/ ijms21031106 
[3] BOEGEMANN M, AYDIN AM, BAGRODIA A, KRABBE LM. Prospects and progress of immunotherapy for bladder cancer. Expert Opin Biol Ther 2017; 17: 1417-1431. https:// doi.org/10.1080/14712598.2017.1366445

[4] KOSHKIN VS, GRIVAS P. Emerging Role of Immunotherapy in Advanced Urothelial Carcinoma. Curr Oncol Rep 2018; 20: 48. https://doi.org/10.1007/s11912-018-0693-y

[5] DUPLISEA JJ, DINNEY C. Should chemotherapy still be used to treat all muscle invasive bladder cancer in the "era of immunotherapy". Expert Rev Anticancer Ther 2019; 19: 543-545. https://doi.org/10.1080/14737140.2019.1625773

[6] CAPALBO L, BASSI ZI, GEYMONAT M, TODESCA S, COPOIU L et al. The midbody interactome reveals unexpected roles for PP1 phosphatases in cytokinesis. Nat Commun 2019; 10: 4513. https://doi.org/10.1038/s41467-01912507-9

[7] VIKBERG AL, VOODER T, LOKK K, ANNILO T, GOLOVLEVA I. Mutation analysis and copy number alterations of KIF23 in non-small-cell lung cancer exhibiting KIF23 over-expression. Onco Targets Ther 2017; 10: 4969-4979. https://doi.org/10.2147/OTT.S138420

[8] JANISCH KM, MCNEELY KC, DARDICK JM, LIM SH, DWYER ND. Kinesin-6 KIF20B is required for efficient cytokinetic furrowing and timely abscission in human cells. Mol Biol Cell 2018; 29: 166-179. https://doi.org/10.1091/ mbc.E17-08-0495

[9] ZHAO C, WANG XB, ZHANG YH, ZHOU YM, YIN Q et al. MicroRNA-424 inhibits cell migration, invasion and epithelial-mesenchymal transition in human glioma by targeting KIF23 and functions as a novel prognostic predictor. Eur Rev Med Pharmacol Sci 2018; 22: 6369-6378. https:// doi.org/10.26355/eurrev_201810_16049

[10] McNeely KC, Cupp TD, Little JN, Janisch KM, Shrestha A et al. Mutation of Kinesin-6 Kif20b causes defects in cortical neuron polarization and morphogenesis. Neural Dev 2017; 12: 5. https://doi.org/10.1186/s13064-017-0082-5

[11] YE L, LI H, ZHANG F, LV T, LIU H et al. [Expression of KIF23 and Its Prognostic Role in Non-small Cell Lung Cancer: Analysis Based on the Data-mining of Oncomine]. Zhongguo Fei Ai Za Zhi 2017; 20: 822-826. https://doi. org/10.3779/j.issn.1009-3419.2017.12.05

[12] SONG X, ZHANG T, WANG X, LIAO X, HAN C et al. Distinct Diagnostic and Prognostic Values of Kinesin Family Member Genes Expression in Patients with Breast Cancer. Med Sci Monit 2018; 24: 9442-9464. https://doi. org/10.12659/MSM.913401

[13] LI XL, JI YM, SONG R, LI XN, GUO LS. KIF23 Promotes Gastric Cancer by Stimulating Cell Proliferation. Dis Markers 2019; 2019: 9751923. https://doi.org/10.1155/2019/9751923

[14] SUN L, ZHANG C, YANG Z, WU Y, WANG H et al. KIF23 is an independent prognostic biomarker in glioma, transcriptionally regulated by TCF-4. Oncotarget 2016; 7: 24646-24655. https://doi.org/10.18632/oncotarget.8261

[15] NONES K, WADDELL N, SONG S, PATCH AM, MILLER $\mathrm{D}$ et al. Genome-wide DNA methylation patterns in pancreatic ductal adenocarcinoma reveal epigenetic deregulation of SLIT-ROBO, ITGA2 and MET signaling. Int J Cancer 2014; 135: 1110-1118. https://doi.org/10.1002/ijc.28765
[16] YANG G, CHANG B, YANG F, GUO X, CAI KQ et al. Aurora kinase A promotes ovarian tumorigenesis through dysregulation of the cell cycle and suppression of BRCA2. Clin Cancer Res 2010; 16: 3171-3181. https://doi.org/10.1158/1078-0432. CCR-09-3171

[17] XYLINAS E, KLUTH LA, RIEKEN M, KARAKIEWICZ PI, LOTAN Y et al. Urine markers for detection and surveillance of bladder cancer. Urol Oncol 2014; 32: 222-229. https://doi. org/10.1016/j.urolonc.2013.06.001

[18] STENZL A, COWAN NC, DE SANTIS M, KUCZYK MA, MERSEBURGER AS et al. Treatment of muscle-invasive and metastatic bladder cancer: update of the EAU guidelines. Eur Urol 2011; 59: 1009-1018. https://doi.org/10.1016/j. eururo.2011.03.023

[19] POWLES T, DURÁN I, VAN DER HEIJDEN MS, LORIOT Y, VOGELZANG NJ et al. Atezolizumab versus chemotherapy in patients with platinum-treated locally advanced or metastatic urothelial carcinoma (IMvigor211): a multicentre, open-label, phase 3 randomised controlled trial. Lancet 2018; 391: 748-757. https://doi.org/10.1016/S01406736(17)33297-X

[20] BELLMUNT J, DE WIT R, VAUGHN DJ, FRADET Y, LEE JL et al. Pembrolizumab as Second-Line Therapy for Advanced Urothelial Carcinoma. N Engl J Med 2017; 376: 1015-1026. https://doi.org/10.1056/NEJMoa1613683

[21] KATO T, WADA H, PATEL P, HU HP, LEE D et al. Overexpression of KIF23 predicts clinical outcome in primary lung cancer patients. Lung Cancer 2016; 92: 53-61. https://doi. org/10.1016/j.lungcan.2015.11.018

[22] SUN X, JIN Z, SONG X, WANG J, LI Y et al. Evaluation of KIF23 variant 1 expression and relevance as a novel prognostic factor in patients with hepatocellular carcinoma. BMC Cancer 2015; 15: 961. https://doi.org/10.1186/s12885-0151987-1

[23] ZHU C, BOSSY-WETZEL E, JIANG W. Recruitment of MKLP1 to the spindle midzone/midbody by INCENP is essential for midbody formation and completion of cytokinesis in human cells. Biochem J 2005; 389: 373-381. https://doi. org/10.1042/BJ20050097

[24] LIN S, LIU M, MOZGOVA OI, YU W, BAAS PW. Mitotic motors coregulate microtubule patterns in axons and dendrites. J Neurosci 2012; 32: 14033-14049. https://doi. org/10.1523/JNEUROSCI.3070-12.2012

[25] BODRUG T, WILSON-KUBALEK EM, NITHIANANTHAM S, THOMPSON AF, ALFIERI A et al. The kinesin-5 tail domain directly modulates the mechanochemical cycle of the motor domain for anti-parallel microtubule sliding. Elife 2020; 9: e51131. https://doi.org/10.7554/eLife.51131

[26] PEI YY, LI GC, RAN J, WEI FX. Kinesin family member 11 contributes to the progression and prognosis of human breast cancer. Oncol Lett 2017; 14: 6618-6626. https://doi. org/10.3892/ol.2017.7053

[27] LI B, LIU B, ZHANG X, LIU H, HE L. KIF18B promotes the proliferation of pancreatic ductal adenocarcinoma via activating the expression of CDCA8. J Cell Physiol 2020; 235: 4227-4238. https://doi.org/10.1002/jcp.29201 
[28] XIE T, LI X, YE F, LU C, HUANG H et al. High KIF2A expression promotes proliferation, migration and predicts poor prognosis in lung adenocarcinoma. Biochem Biophys Res Commun 2018; 497: 65-72. https://doi.org/10.1016/j. bbrc.2018.02.020
[29] TANG Z, LI C, KANG B, GAO G, LI C et al. GEPIA: a web server for cancer and normal gene expression profiling and interactive analyses. Nucleic Acids Res 2017; 45: W98W102. https://doi.org/10.1093/nar/gkx247 